\title{
FUNCIONAMIENTO FAMILIAR DE TRABAJADORES-JEFES DE HOGAR EN SISTEMA DE TURNOS DE UNA EMPRESA DE TALCAHUANO, CHILE, AÑO 2003
}

\author{
FAM ILY M ANAGEM ENT OF HOUSEH OLDER WORKERS IN A \\ SHIFT SYSTEM IN A TALCAHUANO'S ENTERPRISE, CHILE, 2003
}

\author{
JUAN RETAMALES HORMAZÁBAL*, VERÓNICA BEH N THEUNE** \\ YJOSÉ MANUEL MERINO ESCOBAR***
}

\begin{abstract}
RESUMEN
Estudio descriptivo relacional de corte transversal, cuyo propósito fue analizar la percepción del funcionamiento familiar de los trabajadores jefes de hogar en sistemas de turnos rotativo, su pareja y un hijo adolescente.

El universo estuvo constituido por el total de los trabajadores en sistemas de turno de la empresa. La muestra fue 142 familias de trabajadores que reunían las características para el estudio, 59 familias constituyeron la unidad de análisis. Para recolectar los datos se utilizó el instrumento “Cómo es tu familia, cómo es su familia”, de Hernández A.

Los ítemes con significación estadística que resultaron predictores del funcionamiento familiar fueron: Comunicación padre-madre-adolescente, y sus cuatro dimensiones, Afrontamiento de problemas global, Búsqueda de apoyo social, Esfuerzo personal, Poder-dinero y sexo, Satisfacción global, Satisfacción con la vida, y Rendimiento académico/ laboral. Existieron ítemes sin significación estadística pero que pueden constituirse en predictores de riesgo por su condición de déficit.

El funcionamiento familiar de las familias estudiadas es bueno aunque no óptimo. Se destaca como grupo socialmente privilegiado al compararlos con la población media detrabajadores del país, dado sus condiciones económicas, educacionales y culturales, además de un gran apoyo laboral. Los hallazgos indican que en la gran mayoría de los factores existe una tendencia a agruparse en rangos de media-baja vulnerabilidad o riesgo, que implican una baja alteración del funcionamiento familiar.
\end{abstract}

Palabras claves: Funcionamiento familiar, trabajo en turnos, vulnerabilidad o riesgo, familias, trabajadores.

\begin{abstract}
A descriptive relational and cross section study whose aim is to analyze the perception of the family management of householder workers in rotating shift systems, his/her spouse and a teenager son/daughter.

The universe consists of the amount of workers in a plant shift system. The sample was 142 families of workers which had the characteristics for the study, being 59 families considered as analysis unit. Theinstrument used for data collection was the survey "Cómo es tu familia, cómo es su familia", by Hernández A.

The items with statistical significancethat turned out determinant in thefamily management were: Father-M otherAdolescence Communication, and its four dimensions. Facing of Global problems, Searching for Social Support, Selfeffort, Power-M oney and Sex, Global Satisfaction. Life Satisfaction and Academic/Labor Achievement. There were items without statistical significance, but might be determinant of risks due to their deficit condition.

The management in the families who were studied is good but not optimal. It stands out as a socially privilege group when compared to the standard worker groups in the country, due to the economic, educational and cultural conditions, besides a great labor support. The findings show that there is a tendency to group the ranges of medium and low vulnerability or risk in the great majority of factors, which imply a low alteration in the family management.
\end{abstract}

Keywords: Family management, shift work, vulnerability or risk, families, workers.

Recepcionado: 20.11.2003. Aceptado: 28.05.2004

\footnotetext{
*Enfermero, docente Facultad de M edicina, Universidad de Concepción, Concepción-Chile. E-mail: jretamal @udec.cl

**Enfermera, docente Facultad de M edicina, Universidad de Concepción, Concepción-Chile. E-mail: vbehn@udec.cl

***Sociólogo, docenteUniversidad de Concepción, Concepción-Chile. E-mail: jmerino@udec.cl
} 


\section{INTRODUCCIÓN}

La familia es el grupo primario de pertenencia de todas las personas, se considera que es el grupo social más importante en la trama social. La Comisión Nacional dela Familia la define como "un grupo social, unido entre sí por vínculos de consanguinidad, filiación (biológica o adoptiva) y de alianza, incluyendo las uniones de hecho cuando son estables" (1992). La familia cumple múltiples funciones que son básicas para el desarrollo de sus miembros y para la estabilidad dela sociedad, entre ellas está la satisfacción de necesidades biológicas, psicológicas, desocialización, mediadora, y funciones económicas, en esta última es donde sus miembros aprenden a entender la importancia del trabajo.

Se desconoce las implicancias reales que puede tener en la vida de las personas y sus familias desarrollar el trabajo en sistemas de turnos; es así que llama la atención que muchos trabajadores de sistemas deturnos rotativos tengan como aspiración poder llegar a trabajar en horarios diurnos fijos, pero manteniendo los beneficios económicos del trabajar en turnos. Desde hace varios años al gunas empresas chilenas han venido desarrollando dentro de sus políticas laborales las mejores estrategias posibles para mejorar la calidad de vida de sus trabajadores, considerando en forma especial a aquellos que desarrollan su actividad en sistemas deturnos, dado el alto nivel de exigencia que el trabajo demanda y la cantidad de tiempo que permanecen trabajando dentro de la empresa y no junto a su familia.

El funcionamiento familiar consiste en la capacidad del sistema familiar para cumplir con sus funciones esenciales enfrentando y superando cada una de las etapas del ciclo vital, las crisis por las que atraviesa, dando lugar a patrones que permiten ver la dinámica interna en función del medio en que ella se desenvuelve(Espejel y cols., 1997). Para comprender el funcionamiento familiar es necesario analizar su estructura, los procesos y su filosofía familiar. M inuchin, en su Modelo Estructural (1977), definela estructura familiar como "el conjunto invisible de demandas funcionales que organizan los modos en que interactúan los miembros de la familia". Estas pautas o reglas establecen cómo, cuándo, y con quién, cada miembro de la familia se relaciona, regulando la conducta de sus miembros, a su vez refiere que las dimensiones centrales de la estructura familiar son las normas que guían la familia, los roles y las expectativas de rol de cada miembro, los límites, los alineamientos y la jerarquía de poder (M inuchin \& Fischman, 1984). El funcionamiento dela familia se sucede a través de una secuencia de etapas con relación de sentido y continuidad iniciándose con la formación de la pareja y terminando con la muerte de ambos miembros de la pareja. Estas sucesivas etapas por las que atraviesa habitualmente una familia ofrecen numerosos momentos en los cuales pueden aparecer tensiones y conflictos entre sus miembros (Duvall, 1988). Cuando la familia como unidad experimenta un estresor, las tensiones previas se exacerban y las familias las identifican de nuevo como exigencias que deben enfrentar. A pesar dequelos eventos estresantes se presentan en un momento determinado, las tensiones previas pueden no tener un comienzo específico y afectar más insidiosamente a la familia debido justamentea su incierta iniciación; la resolución deestas tensiones es también confusa para la familia y por lo tanto más difícil de afrontar. La acumulación de tensiones y estresores familiares está positivamente asociada con el deterioro del funcionamiento familiar y del bienestar desus miembros. Florenzano(1996) propone hablar de estrés familiar para reconocer quela familia actual se encuentra siempre expuesta a situaciones estresantes, planteando los siguientes indicadores de estrésfamiliar:

- Incapacidad de los miembros para ejercer los roles y tareas usuales.

- Incapacidad para tomar decisiones y resolver problemas. 
- Incapacidades para cuidar a los demás en la forma habitual.

- Cambio de foco de la supervivencia familiar a la supervivencia individual.

El trabajo de turnos es una realidad compleja que posee connotaciones sociales, legalesy económicas que afectan al trabajador ya toda su familia (Roger, Michael y Colligan, 1997; López P., 2001). Diversos estudios subrayan los efectos negativos del trabajo en turnos sobrela vida familiar, queen casos extremos puede volverse total mente desorganizada; como cuando el hombre y la mujer trabajan en turnos diferentes. Pero al margen estos casos, es cierto que la alternancia en los horarios perturba especialmentelos ritmos de vida y las costumbres domésticas: horarios de comidas, momentos de reposo, y momentos de reunión, en fiestas típicas como: Navidades, Semana Santa y acontecimientos familiares. En todos los casos se perturba la organización de la vida familiar, pues el trabajador en turnos se veobligado a adaptar las costumbres de su familia a su horario de trabajo o seguir un ritmo independiente del desu familia (M elo J., 2003).

Basado en lo anterior, el propósito de este estudio fueanalizar la percepción del funcionamiento de las familias de los trabajadoresjefes dehogar en sistemas de turnos rotativos de una empresa, detectando fortalezas y déficits que pudieran constituir predictores de riesgo para la salud de las familias, siendo el objetivo determinar el nivel defuncionamiento familiar de este grupo según la percepción del propio trabajador, su pareja y del hijo adolescente.

\section{MATERIAL Y MÉTODO}

Investigación descriptiva relacional de corte transversal, cuyo universo estuvo constituido por el total detrabajadores en sistemas deturnos rotativos de planta de una empresa de la comuna de Talcahuano contratadosal mes de diciembre2001, totalizando 302 personas. La muestra estuvo constituida por 142 familias que cumplían los criterios de inclusión y superaban los criterios de exclusión, siendo la unidad de análisis el $40 \%$ de estas, realizando la investigación finalmente con 59 familias. Los criterios de inclusión fueron: jefes de hogar varones que trabajen en sistemas de turnos contratados al 31 diciembre 2001 en la empresa, que representen a las distintas áreas de trabajo de turnos, que tengan hijos adolescentes entre 10 y 19 años y pareja estable. $Y$ los criterios deexclusión fueron: M ujeres jefas de hogar, porque ellas están adscritas a sistemas de turnos diurnos fijos, personal que no tenga hijos entre 10 y 19 años, hombres solterosqueno constituyan familia deprocreación, personal contratistatransitorio, personal separado, viudo, anulado, que no cumple con las características de los criterios de inclusión.

La recolección de datos delasfamilias participantes en la investigación se realizó mediante una entrevista domiciliaria, en la que se aplicó una encuesta estandarizada a cada uno de los miembros asignados de la familia denominada "Cómo es tu familia, cómo es su familia", deH ernández A. (1995), quemidela percepción delosmiembros dela familia respecto a sus propias características de funcionamiento familiar, en relación a su estructura, alos procesos deinteracción familiar, eventos estresantes y conductas de riesgos para la salud de los adolescentes y delos adultos. Este Test tiene varias dimensiones que sus autores llaman factores y dentro de ellos hay subfactores. Los factores a saber son: Tipos de Relaciones, Estilo de afrontamiento de los problemas, Fuentes de apoyo del adolescente, Valores, Satisfacción, Acumulación de tensiones, Problemas de salud y de comportamiento, Recursos de la familia. Los puntajes se distribuyen en cinco categorías para cada factor; esas cinco categorías seasocian con el grado devulnerabilidad o riesgo que implica el puntaje obtenido para el funcionamiento familiar. LoS puntajes se distribuyen en una escala que va de mayor a menor vulnerabilidad, la cual se 
ha establecido con base en los resultados del estudio piloto de otros estudios y de las teorías que fundamentan los cuestionarios.

Sesolicitó el consentimiento informado en el momento deaplicar la encuesta, y seinformó sobrela confidencialidad de los datos y el anonimato de los encuestados.

Los datos fueron analizados en el programa computacional SPSS/PC versión-11.0 utilizando estadígrafos descriptivose inferenciales tales como distribución por frecuencias, desviación estándar, t-testy análisisdevarianza.

\section{RESULTADOS}

El perfil dela muestra se representa en la Tabla № 1. Las variables que resultaron predictoras del funcionamiento familiar corresponden a 12 factores y subfactores de entre 32 que se constituían en el instrumento aplicado. Ver Tabla № 2 .

Cabe destacar que en el factor "Tipo de relaciones" hubo una relación estadística altamente significativa en el subfactor comunicación, aún cuando se dimensionó en 4 ca-

Tabla 1. Características sociodemográficas de las familias de trabajadores en sistema de turno.

\begin{tabular}{|l|l|l|c|}
\hline \multirow{2}{*}{$\begin{array}{l}\text { Edad promedio de los } \\
\text { padres y adolescentes }\end{array}$} & Padre & 43,66 años & \multirow{2}{*}{} \\
\cline { 2 - 3 } & Madre & 41,8 años & \multirow{2}{*}{} \\
\cline { 2 - 3 } & Adolescente & 15,15 años & $98,3 \%$ \\
\hline Tipo de familia & Completa & $81,4 \%$ \\
\hline Tamaño de la familia & Entre $4-5$ miembros & $84,7 \%$ \\
\hline Número de hijos & Entre $2-3$ & $66,1 \%$ \\
\hline Etapa de ciclo vital & Adolescente & $93,3 \%$ \\
\hline Educación de los padres & $\begin{array}{l}\text { Secundaria completa a } \\
\text { universitaria completa }\end{array}$ & $81,4 \%$ \\
\hline Trabajadores en la familia & Sólo el padre & $81,4 \%$ \\
\hline Aportantes a los gastos & Sólo el padre & $95 \%$ \\
\hline Acceso a ingresos, bienes y servicios & A todos & . \\
\hline
\end{tabular}

Fuente: Funcionamiento familiar de trabajadores-jefes de hogar en sistema de turnos de una empresa de Talcahuano, Chile, año 2003.

tegorías. En el factor "Afrontamiento de problemas" se encontró relación estadística significativa en el aspecto global y en sólo uno de los cuatro subfactores que la componían, sin constituirse como predictores la búsqueda de apoyo religioso, apoyo profesional y la redefinición del problema. En el factor "Valores" se encontró que el subfactor "Valores morales", no presentando significación estadística, sí es altamente considerado por lo encuestados presentando puntajes cercanosa lo óptimo.

El factor "Satisfacción" sepresenta como un buen predictor del funcionamiento familiar, dado que de cuatro instancias, tres resultaron con relación altamentesignificativa, destacándose que el subfactor "sentimiento de felicidad" aún no siendo predictor se presenta en un nivel medio devulnerabilidad o riesgo para el funcionamiento familiar de estas familias.

Hubo subfactores que, aunque no resultaron ser predictores del funcionamiento familiar, tales como Rutinas familiares, Apoyo del adolescente, esnecesario hacer mención aellos, ya que, por ejemplo, "Rutinas familiares" se encuentra en un nivel medio de vulnerabilidad o riesgo, situación que puede estar relacionada con la instancia de trabajo en turno del padre de familia. En el factor "Apoyo del adolescente" se presenta una situación para- 
Tabla 2. Resumen de variables predictoras del funcionamiento familiar en familias detrabajadores en sistema deturno.

\begin{tabular}{|l|l|l|}
\hline \multicolumn{1}{|c|}{ Factores } & \multicolumn{1}{|c|}{ Subfactores } & Valor - P \\
\hline \multirow{7}{*}{ Tipo de relaciones } & $\begin{array}{l}\text { Comunicación padre/madre } \\
\text { Adolescentes hombres/mujeres }\end{array}$ & 0,000 \\
\cline { 2 - 3 } & $\begin{array}{l}\text { Comunicación padre/madre } \\
\text { Adolescente hombre }\end{array}$ & 0,013 \\
\cline { 2 - 3 } & $\begin{array}{l}\text { Comunicación padre/madre } \\
\text { Adolescente mujer }\end{array}$ & 0,002 \\
\cline { 2 - 3 } & $\begin{array}{l}\text { Comunicación padre/madre } \\
\text { Adolescente entre 10-14 años }\end{array}$ & 0,001 \\
\cline { 2 - 3 } & $\begin{array}{l}\text { Comunicación padre/madre } \\
\text { Adolescente entre 15-19 años }\end{array}$ & 0,016 \\
\hline \multirow{2}{*}{ Afrontamiento de } & & 0,016 \\
\hline problemas global & Búsqueda de apoyo social & 0,000 \\
\hline Valores & $\begin{array}{l}\text { Esfuerzo personal } \\
\text { Poder, dinero y sexo }\end{array}$ & 0,000 \\
\hline Satisfacción global & Satisfacción con la vida & 0,000 \\
\hline \multirow{2}{*}{} & Rendimiento académico/laboral & 0,000 \\
\hline
\end{tabular}

Fuente: Funcionamiento familiar de trabajadores-jefes de hogar en sistema de turnos de una empresa de Talcahuano, Chile, año 2003.

dójica al encontrar quela percepción del adolescente tiene puntajes más al tos que la percepción de ambos padres acerca de su hijo.

\section{DISCUSIÓN}

Se puede decir que el funcionamiento familiar de las familias estudiadas es bueno aunque no óptimo, al comparar los factores que integran el instrumento "Como es su familia y como es tu familia" para evaluar el funcionamiento familiar. Es necesario destacar que esun grupo socialmente privilegiado al compararlos con la población media de trabajadores del país, dado que ellos cuentan con un nivel económico, educacional y cultural fuerte, además de un gran apoyo laboral y también intrafamiliar queles permiten tener conductas que se diferencian de los grupos promedios de trabajadores siendo ésta la condi- ción que le da ventajas sobre otras familias. Es probable que esto explique la similitud en los puntajes promedios obtenidos tanto para padres, madres y adolescentes en cada uno de los factores y subfactores estudiados.

Loshallazgosindican que, si bien en la gran mayoría de los factores existe una tendencia a agruparse en los rangos de media baja vulnerabilidad o riesgo queimplican una baja alteración del funcionamiento familiar, se puede apreciar que en los grupos de padres, madres y adolescentes existen diferencias estadísticamente significativas, destacándose dentro de los predictores el subfactor "Comunicación", observándose una tendencia a una buena relación delos padres con hijos hombres y mujeres, dentro de todo el rango de edad de los adolescentes estudiados (entre 10 y 19 años).

El estudio deFlorenzano y cols. (1996) coincidecon los resultados deesta investigación, al considerar que, a mayores ingresos fami- 
liares, baja la probabilidad de riesgo; también se da que, a mayor nivel de estudios y que la situación de trabajo sea estable, existe menor nivel de riesgo o vulnerabilidad en el funcionamiento familiar. Asimismo, el factor "Afrontamiento de problemas" presenta niveles de riesgo al tos, dado que en las familias estudiadas no utilizan las redes de apoyo social, religioso y/o profesional, lo que puede estar relacionado con la excesiva confianza en la redefinición interna de sus problemas; este último se presenta con un alto puntaje, probablementeesta característica delasfamilias esté inducida por la preocupación de la imagen social que ellos mantienen. En tanto que, al relacionar la cantidad de proveedores, se presenta una relación inversa entre ambos estudios, dado que en la presente investigación el $80 \%$ de las familias posee un proveedor económico y bajo nivel de riesgo o vulnerabilidad en relación con el funcionamiento familiar, contrario a lo que presenta el estudio de Florenzano y cols. Cabe destacar que el proveedor de estas familias aporta en general tres o más veces quelo aportado por lo proveedores estudiados en las familias del estudio de Florenzano.

Se aprecia claramenteen la presenteinvestigación que en estas familias la cohesión familiar, participación, comunicación padre/ madre-hijo/a y comunicación conyugal son características de familias saludables. Se puede destacar que las creencias religiosas, siendo un rasgo de las familias saludables y estando presente en las familias estudiadas, no se constituye como una oportunidad para apoyar la resolución de problemas, lo que coincide con lo planteado por Currant (1983), Chamblás y cols. (2000).

Secorroboralo señalado por Roger, M ichael y Colligan (1997), López (2001) y M elo (2003) al plantear efectos negativos en la vida social y familiar del trabajo en turnos, situación que en esta investigación se presenta con un nivel medio devulnerabilidad o riesgo en el subfactor "Rutinas familiares", lo que implica que disponen depocosmomentos compartidosen familia. Lo anterior es reforzado por Florenzano y cols. (1996), Arriagada y Cuevas (2003) al señalar que hay factores que hacen más compleja la convivencia familiar y destaca entreellos la mayor permanencia en el trabajo, afectando la permanencia en el hogar y los tiempos de interacción familiar.

En el factor "Valores" se pudo evidenciar que estas familias tienen una gran valoración respecto a alcanzar altos niveles de estudio y sentirse realizado en el trabajo, como a su vez le asignan una alta significación a los valores personales, representados por el respetar a los demás y ser respetado, ser una persona honesta y recta, creer en Dioso un ser superior, tener una familia unida, lo que es coincidente con el estudio de Chamblás (2000).

El nivel deSatisfacción global, Satisfacción con la vida, y Rendimiento académico/laboral de los miembros de la familia tienen directa relación con el funcionamiento familiar. Sin embargo, se detecta al gunos aspectos contrastados, evidenciados por el hecho de sentirse satisfechos con la vida, asociado al poder contar con servicios de salud, con una situación económica estable y servicios en general; no así en el subfactor Rendimiento académico/ laboral donde sólo el padrey el adolescentereconocen estar satisfechos como la mayoría, no así la madre que reconoce abiertamente estar insatisfecha lo que se refleja en muy bajos puntajes promedios (1,14 puntos de una escala de 0 a 5 puntos). La insatisfacción laboral encontrada en la madre pudiera explicarse por el hecho que la madre sólo se desarrolla como dueña de casa y está limitada en su desarrollo social y de expectativas. Este planteamiento se asocia fuertemente con lo señalado por Parsons (1967) y Florenzano (1996) al plantear que el estatus de una persona se relaciona con la percepción de la importancia que tiene su rol y el prestigio que le proporciona, dado que en el contexto familiar actual se evidencia un progresivo alejamiento deloshijosy una menor necesidad decuidado exclusivo de parte de la madre, quedando en consecuencia mayor tiempo sola. 
En relación con las conductas de riesgos de estas familias, como el consumo de cigarrillos, el uso de drogas tranquilizantes y el inicio de actividad sexual en una pequeña proporción de los adolescentes, se puede decir que son de muy baja incidencia y se constituyen en factores protectores familiares que deben ser reforzadosa nivel educacional y laboral.

Cada país o sociedad se conoce cuando se estudia cómo vive y actúa la familia, si queremos prever el futuro de una sociedad podemos saber bastante de ella observando las familias, si queremos reflexionar sobre la identidad personal, por qué somos de una forma y no de otra, por quéactuamos de esta manera, por qué nos orientamos en determinado sentido, gran parte de esta respuesta está en la historia familiar decada uno. Ello nos debe hacer reflexionar acerca de la importancia de la familia para el individuo, para la sociedad y en especial para la calidad de vida de las familias.

Por la importancia que tiene en la salud y la calidad de vida de las familias, de los trabajadoresque desarrollan su trabajo en sistemas deturnos, es quela enfermería en salud laboral tieneuna gran labor a desarrollar; para esto debe reconocer en esta área una de las que enfermería no ha desarrollado mucha investigación, esto se corrobora con las escasas investigaciones encontradas realizadas por enfermeras/os.

Otro de los fines principales de cada país debe ser incrementar el nivel de salud de la población, haciendo referencia a la familia como uno de los grupos fundamentales en el terreno de la salud y es aquí donde enfermería desarrolla su quehacer diariamente, por esto resulta fundamental el desarrollo de la investigación en los procesos familiares.

Lo antes expuesto me hace reflexionar sobrela importancia deplanificar estrategias de intervención dirigidas a promover una adecuada salud familiar. La intervención psicológica familiar consisteen la aplicación de un conjunto de acciones a través de las cuales la familia desarrolle sus propios recursos psicológicos, se propicie la autoayuda, facilitando queella demanera independientesea capaz de hallar soluciones adaptativas ante situaciones conflictivas y de crisis que se presentan en la vida cotidiana, tarea que deben desarrollar los profesionales deenfermeríaque participan en los programas de salud mental de los consultorios o centros de salud familiar.

La necesidad deintervenir a la familia viene dada por la importancia de este grupo en el desarrollo de la personalidad de sus miembros. Lasmodalidades deintervención con las familias dela comunidad generalmenteestán basadas en metodologías grupales, cuyo fin es la búsqueda de la reflexión y análisis para encontrar soluciones alternativas ante los diversos problemas o situaciones transitorios que presenta la familia en el desarrollo de su ciclo vital. Considero que estas estrategias de intervención en la comunidad deben convertirse en herramientas de trabajo del equipo de enfermería con el fin de promover modos y estilos de vida favorables a la salud familiar y a su vez un gran campo deinvestigación que permita tener nuevo conocimiento queapunte a mejorar la vida familiar.

\section{REFERENCIAS BIBLIOGRÁFICAS}

ARRIAGADA, O. y CUEVASL. (2003). Calidad devida de grupos familiares, dependientes de un jefe de hogar que labora en modalidad de turno rotativo o de alta permanencia. Concepción, Chile, tesis de pregrado Servicio Social Universidad de Concepción.

CH AM BLÁS, I.; M ATHIESEN, M .E.; MORA, O.; NAVARRO, G.; CASTRO, M . (2000). "Funcionamiento familiar. Una mirada desde los hijos/estudiantes deenseñanza media dela provincia deConcepción", Concepción, Chile, Revista electrónica de Trabajo Social Universidad de Concepción.

COMISIÓN NACIONAL DE LA FAMILIA (1992). Servicio Nacional de la Mujer: Informe Comisión Nacional de la Familia. Chile.

CURRANT D. (1983). Traits of healthy family. Minneapolis. Ed. Winston.

DUVAL E.M . (1977). Marriage and family development. Filadelfia. Lippincott. 
DUBALL E. (1988). Aplicación del resultado de la pesquiza". Machado Lopez.

ESPEJEL, E. (1997). Estudios defamilia en relación con la esquizofrenia. Instituto de la Familia. M éxico.

FLORENZANO, Ramón (1996). Familia y salud delos jóvenes. Santiago, Chile: Ediciones Universidad Católica de Chile.

HERNÁNDEZ A. (1996). Familia y adolescencia: Indicadores de salud. M anual de aplicación de instrumentos. Ed. F. Kellogg. OPS.

HIDALGO, C. y CARRASCO, E. (1999). Salud familiar: Un modelo deatención integral en la atención primaria. Santiago, Chile: Ediciones Universidad Católica de Chile.

LÓPEZ P. (2001). Percepción de factores laborales de las mujeres que se desempeñan en trabajo de turno. Concepción, Chile, tesis de pregrado Servicio Social Universidad de Concepción.

M ELO, José Luis (2003). “Sistemas de trabajo por turnos". Argentina. www.estrucplan.com.ar, 27 de junio.

M INU CH IN S. (1977). Familias y terapia familiar. España. Ed. Gedisa.

M IN U CHIN S. Fischman H. (1984). Técnicas deterapia familiar. Buenos Aires: Ed. Paidós.

PARSON S, Talcott (1967). Ensayos de teoría sociológica. Buenos Aires, Argentina: Editorial Paidós.

RO GER R., Michael R. Colligan J. (1997). El trabajo por turnos en lenguaje sencillo. Departamento de Salud y Servicios Humanos de losEE.UU. (traducción en español), agosto 2002. 